\title{
On the Capacity Region of Multi-Antenna Gaussian Broadcast Channels with Estimation Error
}

\author{
Amir F. Dana, Masoud Sharif, Babak Hassibi \\ Department of Electrical Engineering, \\ California Institute of Technology \\ Pasadena, CA 91125, USA \\ Email: \{dana, hassibi\}@systems.caltech.edu, sharif@bu.edu
}

\begin{abstract}
In this paper we consider the effect of channel estimation error on the capacity region of MIMO Gaussian broadcast channels. It is assumed that the receivers and the transmitter have (the same) estimates of the channel coefficients (i.e., the feedback channel is noiseless). We obtain an achievable rate region based on the dirty paper coding scheme. We show that this region is given by the capacity region of a dual multi-access channel with a noise covariance that depends on the transmit power. We explore this duality to give the asymptotic behavior of the sum-rate for a system with a large number of user, i.e., $n \rightarrow \infty$. It is shown that as long as the estimation error is of fixed (w.r.t $n$ ) variance, the sum-capacity is of order $M \log \log n$, where $M$ is the number of antennas deployed at the transmitter. We further obtain the sum-rate loss due to the estimation error. Finally, we consider a training-based scheme for block fading MISO Gaussian broadcast channels. We find the optimum length of the training interval as well as the optimum power used for training in order to maximize the achievable sum-rate.
\end{abstract}

\section{INTRODUCTION}

There has been recently a great deal of research on the capacity region of the MIMO Gaussian broadcast channels (e.g. see [1][5]). These channels are of practical importance since they can be used as a model for the down-link of cellular systems. In [5], the authors show that the entire capacity region is achieved by an interference pre-substraction coding scheme known as dirty paper coding (DPC) first introduced in [6].

While DPC is the optimal transmission scheme, it is computationally expensive and also requires the transmitter to have perfect knowledge of the channel state information for all the users. Furthermore, the capacity of broadcast channels highly depends on the amount of channel state information in the transmitter (CSI). If there is no CSI available at the transmitter employing multiple antennas does not increase the throughput significantly. However when perfect CSI is available the throughput scales linearly with the number of transmit antennas (as the transmit power or the number of users increases). From a practical point of view, simple and effective scheduling schemes that are robust against noisy channel state information (and/or require partial knowledge of the channel) and also have a good performance are desirable [21]. There has been some progress on devising simple scheduling schemes that operate close to boundary points of the capacity region with limited feedback [15], [16], [20], [18].

\footnotetext{
${ }^{1}$ This work is supported in part by the National Science Foundation under grant nos. CCR-0133818 and CCR-0326554, by the David and Lucille Packard Foundation, and by Caltech's Lee Center for Advanced Networking.
}

However, the requirement of having accurate channel estimation is a strict constraint.

In this paper we consider the effect of channel estimation error on the capacity of MIMO Gaussian broadcast channels. We propose an achievable region based on the dirty paper coding scheme. This scheme is essentially similar to the achievable rates obtained for MIMO point to point and multi-access channels with uncertainty in channel measurements [12], [13]. We further show a duality between the achievable rate region and the capacity of a multi-access channel where the noise covariance is dependent on the transmit power at different users. This duality is explored to show the effect of the estimation error on the sum-rate for large number of users. It is shown that as long as the estimation error is fixed with respect to the number of users, we achieve the same scaling law as if there was no estimation error. Of course, there is a loss due to the estimation error in the sum-rate which is obtained as a function of the variance of the estimation error. Based on the achievable rate region derived earlier, we analyze the performance of a training-based scheme for block fading models. We show that the optimal amount of time used for training is equal to the number of transmit antennas.

\section{System MOdEL}

We consider a block fading Gaussian MIMO broadcast channel with channel estimation error. The transmitter employs $M$ transmit antennas. We assume that there are $n$ users in the system each equipped with $r_{i}, i=1, \ldots, n$ antennas. The channel matrix between the transmitter and user $i$ is an $M \times r_{i}$ matrix and is denoted by $H_{i}$. A block fading model with coherence interval of length $T$ is considered. We assume that the channel coefficients for each user are zero mean jointly Gaussian random variables with covariance matrix $\operatorname{cov}\left(H_{i}\right)=\mathrm{E}\left(\operatorname{vec} H_{i}\right)\left(\operatorname{vec} H_{i}\right)^{*}=R_{H}$. The received signal at user $i$ is given by

$$
y_{i}=H_{i} x+n_{i}
$$

where $n_{i}$ is additive white Gaussian noise with zero mean and identity covariance matrix. $x$ is the input vector with power constraint $\mathrm{E}\left[x^{*} x\right] \leq P$.

In this paper, the users and the transmitters do not have exact knowledge of the channel matrices. We assume that user $i$ estimates its channel to $\hat{H}_{i}$. This estimate is fed back to the transmitter through a perfect channel. The channel estimation error $\tilde{H}_{i}$ which is equal to $H_{i}-\hat{H}_{i}$ is assumed to be uncorrelated from the estimate $\hat{H}_{i}$ (i.e, MMSE estimation). The coordinates 
of $\tilde{H}_{i}$ are assumed to be jointly Gaussian random variables with covariance matrices of form $A_{i}^{T} \otimes K_{i}$, where $A_{i}$ and $K_{i}$ are positive semi-definite $M \times M$ and $r_{i} \times r_{i}$ matrices. The above covariance matrix models the possible correlation at the transmitter and the receiver side (see [19]).

The capacity region of the aforementioned broadcast channel is known when $H_{i}$ is available to the transmitter and to the $i$ 'th receiver for $i=1, \ldots, n$ [5]. Assuming the noise covariance matrix $N_{i}$ for the $i$ 'th user, and under the transmit covariance matrix constraint, i.e., $\mathrm{E}\left[x x^{*}\right] \preceq S$, the capacity region is given by dirty paper coding and can be written as

$$
\mathcal{C}\left(S,\left\{N_{i}\right\},\left\{H_{i}\right\}\right)=\mathrm{conv} \bigcup_{\pi,\left\{B_{i}\right\}} \mathcal{R}\left(\pi, S,\left\{B_{i}\right\},\left\{N_{i}\right\},\left\{H_{i}\right\}\right)
$$

where the union is over all permutations on set $\{1, \ldots, n\}$ and all positive semi-definite covariance matrices $B_{1}, \ldots, B_{n}$ such that $\sum_{i=1}^{n} B_{i} \preceq S$ and

$$
\begin{aligned}
& \mathcal{R}\left(\pi, S,\left\{B_{i}\right\},\left\{N_{i}\right\},\left\{H_{i}\right\}\right)=\left(R_{1}, \ldots, R_{n}\right) \\
& 0 \leq R_{\pi(i)} \leq \log \frac{\left|N_{\pi(i)}+H_{\pi(i)}\left(\sum_{k=1}^{i} B_{\pi(k)}\right) H_{\pi(i)}^{*}\right|}{\left|N_{\pi(i)}+H_{\pi(i)}\left(\sum_{k=1}^{i-1} B_{\pi(k)}\right) H_{\pi(i)}^{*}\right|}
\end{aligned}
$$

Finally, the capacity region of the broadcast channel with average total transmit power constraint $P$, i.e., $\operatorname{Tr}(S) \leq P$, is given by the

$$
\mathcal{C}\left(P,\left\{N_{i}\right\},\left\{H_{i}\right\}\right)=\bigcup_{S: \operatorname{Tr}(S) \leq P} \mathcal{C}\left(S,\left\{N_{i}\right\},\left\{H_{i}\right\}\right) .
$$

In order to compute any point on the boundary of the capacity region, [2], [3] establish a duality between the capacity region of broadcast and multiple access channels under sum power constraints. This duality is considered in a more general scenario and based on the mini-max (and the Lagrangian) duality in [7], [8]. These results are very useful since the multi-access channel capacity region is much easier to compute [9].

\section{INNER-BOUND ON THE CAPACITY REGION}

In this section we give an inner bound on the capacity region of the Gaussian MIMO broadcast channel with estimation error. The results are based on the fact that the worst uncorrelated noise with given covariance matrix has Gaussian distribution. This was in fact used previously to obtain lower bounds on the capacity of MIMO point-to-point channels and multi-access channels in [12], [13].

Theorem 1: Consider a Gaussian MIMO broadcast channel described in section II where the estimated channel for the $i$ 'th user is $\hat{H}_{i}$ which is known to the transmitter and the corresponding user. The capacity region then includes the capacity region of a MIMO Gaussian broadcast channel with channel matrices $\hat{H}_{i}$ and effective noise covariance $I+\operatorname{Tr}\left(A_{i}\left(\sum_{l=1}^{n} B_{l}\right)\right) K_{i}$. In other words, capacity region includes

$$
\text { conv } \bigcup_{\substack{\operatorname{Tr}\left(\sum_{i},\left\{B_{i}\right\} \\ B_{i}\right) \leq P}} \mathcal{R} \pi, S,\left\{B_{i}\right\},\left\{I+\operatorname{Tr}\left(A_{i}\left(\sum_{l=1}^{n} B_{l}\right)\right) K_{i}\right\},\left\{\hat{H}_{i}\right\}
$$

Sketch of proof: The proof follows using the dirty paper coding scheme. Suppose that $x_{1}, \ldots, x_{n}$ are independent Gaussian vectors with zero mean and covariance $B_{1}, \ldots, B_{n}$ that are generated according to a dirty paper coding scheme with an order according to permutation $\pi$ (here we assume the identity permutation) and that $x=\sum_{i=1}^{n} x_{i}$ is the transmitted signal. The received signal at user $i$ can be written as

$$
y_{i}=\hat{H}_{i} x_{i}+\hat{H}_{i}\left(\sum_{j=i+1}^{n} x_{j}\right)+\underbrace{\hat{H}_{i}\left(\sum_{j=1}^{i-1} x_{j}\right)+\tilde{H}_{i}\left(\sum_{i=1}^{n} x_{i}\right)+n_{i}}_{v_{i}}
$$

Now using the result of [10] on the capacity of memoryless channels with random state known non-causally by the transmitter, we can show that the following rate is achievable for user $i$ in (1)

$$
R_{i}=I\left(u_{i} ; y_{i}\right)-I\left(u_{i} ; s_{i}\right)
$$

where the random state is $s_{i}=\sum_{j=i+1}^{n} x_{j}$ and is known by the encoder and the channel input is $x_{i}$. Also $u_{i}$ has a Gaussian distribution and is a function of $\hat{H}_{i}, x_{i}, x_{i+1}, \ldots, x_{n}$. The second mutual information term in (2) does not depend on the distribution of $\tilde{H}_{i}$. The first term in (2) can be written as

$$
I\left(u_{i} ; y_{i}\right)=h\left(u_{i}\right)-h\left(u_{i} \mid y_{i}\right)
$$

We have the following bound on $h\left(u_{i} \mid y_{i}\right)$

$$
h\left(u_{i} \mid y_{i}\right) \leq \log \left|\pi e \times \operatorname{cov}\left(u_{i} \mid y_{i}\right)\right|
$$

since, for a given covariance matrix, the Gaussian distribution has the largest entropy.

Therefore, looking at (1), by considering $v_{i}$ to be a Gaussian random vector with zero mean and the same covariance matrix we get a lower bound on $R_{i}$ in (2). The covariance of $v_{i}$ is

$$
\mathrm{E}\left(v_{i} v_{i}^{*}\right)=\hat{H}_{i}\left(\sum_{j=1}^{i-1} B_{j}\right) \hat{H}_{i}^{*}+\mathrm{E} \tilde{H}_{i}\left(\sum_{j=1}^{n} B_{j}\right) \tilde{H}_{i}^{*}+I
$$

The first term on the right hand side is the contribution from the part of interference that is treated as noise. The second term comes from the error in estimating the channel. It can be shown that for $\tilde{H}_{i}$ with $\operatorname{cov}\left(\tilde{H}_{i}\right)=A_{i}^{T} \otimes K_{i}$ we have

$$
\mathrm{E}\left(\tilde{H}_{i}\left(\sum_{l=1}^{n} B_{l}\right) \tilde{H}_{i}^{*}\right)=\sum_{l=1}^{n} \operatorname{Tr}\left(B_{l} A_{i}\right) K_{i} .
$$

Therefore the effective noise of the system has covariance

$$
\sum_{l=1}^{n} \operatorname{Tr}\left(B_{l} A_{i}\right) K_{i}+I
$$

Having this, we get the rate region given in the theorem statement.

\section{Optimal Power Allocation}

In the previous section an achievable rate region for MIMO broadcast channels with estimation error was given. This region is based on dirty paper coding. It is well known that the dirty paper coding region is not convex in input covariance matrices and finding the boundary points of the capacity region directly from the dirty paper coding regime is not computationally tractable. However, using the duality of the broadcast and multiple access channels [2], [3], and the mini-max duality introduced in [7], [8] it is possible to find the boundary points of the capacity region under some class of power constraints using convex optimization. In this section we consider finding the power allocation for any boundary point on the achievable rate region described in Theorem 1. 
It is worth mentioning that since in our case the effective noise covariance matrix also depends on the input covariance matrices, it can be verified that the transformations used in [3] do not go through ${ }^{1}$. For the presentation of this paper we provide duality results in the following two cases.

1) For all users $A_{i}=I$ : It can be easily shown that any boundary point on the region described in Theorem 1 is achieved when $\sum_{i=1}^{n} \operatorname{Tr}\left(B_{i}\right)=P$. Therefore if $A_{i}=I$ for all the channels, the effective noise of (3) does not depend on $B_{i}$ anymore and is given by $I+P K_{i}$. In this case, one can use the duality of multiple access and broadcast channels with sum power constraints. Hence the region of Theorem 1 is equal to the capacity region of a Gaussian Multiple access channel with sum power constraint $P$ and channel coefficients $\hat{H}_{i}^{*}\left(I+P K_{i}\right)^{-\frac{1}{2}}$. Therefore, any point on the boundary can be computed using convex optimization. We have summarized this result as follows.

Theorem 2: Consider the setting of Theorem 1. Further assume that the covariance matrix of the estimation error for $i$-th channel is of the following form $\operatorname{cov}\left(\tilde{H}_{i}\right)=I \otimes K_{i}$ then the capacity region of the channel includes the capacity region of a multiple access channel with sum power constraint $P$ and channel coefficients $\hat{H}_{i}^{*}\left(I+P K_{i}\right)^{-\frac{1}{2}}$.

2) MISO broadcast with estimation error, i.e. $r_{i}=1:$ In the rest of this paper, we consider the achievable rates for MISO broadcast channels with estimation error. For this case, we can state the achievable region based on the capacity region of a dual multiple access channel.

Theorem 3: Consider a MISO Gaussian broadcast channel with estimation error covariance $A_{i} \succ 0$ for user $i$ and total transmit power constraint of $P$. Then, the capacity region includes the capacity region of a multiple access channel with one antenna at each transmitter and $M$ antennas at the receiver. The channel coefficient vector for transmitter $i$ is $\hat{H}_{i}^{*}$. The total transmit power constraint is $P$ and the noise covariance is $I+\sum_{i=1}^{n} P_{i} A_{i}$ where $P_{i}$ is the transmit power for user $i$.

Proof: Sketch of the proof of the above theorem is provided in Appendix A.

Clearly, Theorem 3 implies that for a homogeneous system, where $A_{i}=A$ for all users, the capacity region of this channel includes the capacity region of a multiple access channel with total transmit power $P$ and noise covariance matrix $I+P A$.

\section{Scaling Laws of the AChievable sum-Rate}

Using Theorem 3, we know that the following sum-rate is achievable for homogeneous MISO broadcast channels.

$$
R_{\text {sum }}=\max _{\substack{P_{i} \geq 0 \\ \sum_{i=1}^{n} P_{i} \leq P}} \log \frac{\left|I+P A+\sum_{i=1}^{n} P_{i} \hat{H}_{i}^{*} \hat{H}_{i}\right|}{|I+P A|}
$$

This optimization problem is convex in the $P_{i}$ 's and can be therefore solved when $n$ is not too large. The achievable ergodic sum-rate for fading channels is just the expectation of $R_{\text {sum }}$ over

\footnotetext{
${ }^{1}$ The transformation used in [3] is valid for a sum power constraint.
}

all channel realizations. Clearly when $n$ is large, computing the average sum-rate becomes computationally intensive. In what follows, we obtain the scaling law of the ergodic sum-rate for large number of users. by

Defining $G_{i}=\hat{H}_{i}(I+P A)^{-\frac{1}{2}}$, the ergodic sum-rate is given

$$
R^{\star}=\mathrm{E}\left(R_{\text {sum }}\right)=\mathrm{E} \max _{P_{i} \geq 0, \sum_{i=1}^{n} P_{i} \leq P} \log \left|I+\sum_{i=1}^{n} P_{i} G_{i}^{*} G_{i}\right|
$$

where the expectation is over $G_{i}$ 's for $i=1, \ldots, n$. Here $G_{i}$ 's are independent Gaussian vectors with covariance matrix

$$
\mathrm{E}\left(G_{i}^{*} G_{i}\right)=(I+P A)^{-\frac{1}{2}}\left(R_{H}-A\right)(I+P A)^{-\frac{1}{2}} .
$$

Note that (5) is in fact the ergodic sum-capacity of a MISO broadcast channel where channels are distributed according to a Gaussian distribution with covariance matrix given in (6). The ergodic sum-capacity of MISO broadcast channel with spatial correlation in channel coefficients is analyzed for large number of users in [11]. Assuming that $R_{H}$ and $A$ are fixed (in terms of $n$ ), one can use the result of [11] to state the following Theorem.

Theorem 4: Consider the setting of Theorem 3. Assume the channel covariance matrix is $R_{H}$ and estimation error covariance is $R_{H} \succeq A$. Then as the number of users $n$ goes to infinity the achievable sum-rate scales like

$$
\begin{aligned}
R^{\star}= & M \log \log n+M \log \frac{P}{M} \\
& +\log \operatorname{det}\left(R_{H}-A\right)-\log \operatorname{det}(I+P A)+o(1)
\end{aligned}
$$

Theorem 4 suggests that as long as the estimation error covariance matrix is fixed in terms of $n$, one gets the same scaling as the case where the channel is known perfectly at the receivers and the transmitter. In fact, the effect of estimation error shows up as a constant hit in the achievable rate.

At the end, We should remark that since for a homogeneous network, the equivalent noise in Theorem 3 is linear in the transmit power, in the high SNR regime (and for a fixed number of users), the achievable sum-rate will be of constant order (See also [17]).

\section{TRAINING}

The results obtained so far are based on a given estimation error covariance. To estimate the channel, a training phase is often required. During this phase, some portion of the transmission interval and transmit power is used to send known training signals. In this section we consider training for block fading MISO broadcast channels with $M$ transmit antennas, coherence interval of $T \geq M$ and total transmit power of $P$. We further assume that the channel coefficients are independent zero mean unit variance Gaussian random variables. We find the optimum amount of time and power that should be allocated for training to maximize our achievable sum-rate.

During the training phase, the transmitter send $T_{\tau}$ training vectors with total transmit energy of $P_{\tau} T_{\tau}$. Let $X_{\tau}$ be the $M \times T_{\tau}$ matrix consisting of the training vectors. We have

$$
\operatorname{Tr}\left(X_{\tau}^{*} X_{\tau}\right)=P_{\tau} T_{\tau}
$$

The received signal at user $i$ can be written as

$$
y_{i, \tau}=h_{i} X_{\tau}+v_{i, \tau}
$$


At the end of the training phase, each user finds the LMMSE estimate of its channel and feeds it back to the transmitter. In order to obtain a meaningful estimate of $h_{i}{ }^{2}$, we need at least as many measurements as unknown, which implies that $T_{\tau} \geq M$. The estimate can be written as

$$
\hat{h}_{i}=y_{i, \tau}\left(I+X_{\tau}^{*} X_{\tau}\right)^{-1} X_{\tau}^{*}
$$

Note that $y_{1, \tau}, \ldots, y_{n, \tau}$ are independent and identically distributed. The estimation error covariance for every user is

$$
\begin{aligned}
A_{\tau}=\operatorname{cov}\left(\tilde{h}_{i}\right) & =I-X_{\tau}^{*}\left(I+X_{\tau}^{*} X_{\tau} X_{\tau}\right)^{-1} X_{\tau} \\
& =\left(I+X_{\tau} X_{\tau}^{*}\right)^{-1}
\end{aligned}
$$

Let $T_{d}=T-T_{\tau}$ and $P_{d} T_{d}=P T-P_{\tau} T_{\tau}$. After the training phase, the transmitter starts sending data over the $T_{d}$ time samples left and with total transmitter energy $P_{d} T_{d}$. Therefore for a fixed $P_{\tau}, T_{\tau}$, using the result of Theorem 3 , the following sum-rate is achievable

$$
R_{\tau}=\frac{T_{d}}{T} \mathrm{E} \max _{P_{i} \geq 0, \sum_{i} P_{i} \leq P_{d}} \log \frac{\left|I+P_{d} A_{\tau}+\sum_{i=1}^{n} P_{i} \hat{h}_{i}^{*} \hat{h}_{i}\right|}{\left|I+P_{d} A_{\tau}\right|},
$$

where $h_{i}$ s are independent vectors whose elements are jointly Gaussian random variables with covariance matrix $I-A_{\tau}$ (which follows from the orthogonality principle). Now consider the eigenvalue decomposition of $X_{\tau} X_{\tau}^{*}=U \Omega U^{*}$, where $U$ is unitary and $\Omega$ is diagonal and from $(8)$ we have $\operatorname{Tr}(\Omega) \leq P_{\tau} T_{\tau}$. After some manipulation of (9) we can rewrite the achievable rate as

$$
R_{\tau}=\frac{T_{d}}{T} \mathrm{E} \max _{\substack{P_{i} \geq 0 \\ \sum_{i} P_{i} \leq P_{d}}} \log \frac{\left|I+\left(1+P_{d}\right) \Omega^{-1}+\sum_{i=1}^{n} P_{i} g_{i}^{*} g_{i}\right|}{\left|I+\left(1+P_{d}\right) \Omega^{-1}\right|} .
$$

The $g_{i}$ 's are independent vectors whose elements are independent zero mean unit variance Gaussian random variables and the expectation is over $g_{i}$. Now let us consider the case where $\Omega$ is a scaled version of identity. Using the trace constraint we have

$$
\Omega=\frac{P_{\tau} T_{\tau}}{M} I
$$

This $\Omega$ corresponds to the case where the training matrix $X_{\tau}$ is a multiple of a matrix with orthonormal columns. Also it can be shown that this choice of $\Omega$ corresponds to the worst case noise in (10), i.e.,

$$
\frac{P_{\tau} T_{\tau}}{M} I=\underset{\Omega, \operatorname{Tr}(\Omega) \leq P_{\tau} T_{\tau}}{\operatorname{argmin}} R_{\tau}
$$

Using this $\Omega$ and simplifying (10), the following rate is achievable

$$
R_{\tau}=\frac{T_{d}}{T} \mathrm{E} \max _{P_{i} \geq 0, \sum_{i} P_{i} \leq P_{\text {eff }}} \log \left|I+\sum_{i=1}^{n} P_{i} g_{i}^{*} g_{i}\right|
$$

where for each $i, g_{i}$ is a vector of i.i.d zero mean unit variance Gaussian random variables. $P_{\text {eff }}$ is the effective power and is given as

$$
P_{\mathrm{eff}}=\frac{P_{d} P_{\tau} T_{\tau}}{P_{\tau} T_{\tau}+\left(1+P_{d}\right) M} .
$$

We can maximize the achievable lower bound of (11) over power and time allocated for training. Note that for a fixed $T_{\tau}$ (and $T_{d}$ ),

\footnotetext{
${ }^{2}$ Throughout this section we use $h_{i}$ rather than $H_{i}$ to represent the channe vector for $i$-th user.
}

the optimal power allocation is one that maximizes the effective transmit power $P_{\text {eff }}$. By maximizing $P_{\text {eff }}$ over $P_{\tau}$ and $P_{d}$ we get

$$
P_{\text {eff }}^{\star}\left(T_{d}\right)=\frac{(P T)^{2}}{\sqrt{\left(P T+T_{d}\right) M}+{\sqrt{(M+P T) T_{d}}}^{2}} .
$$

Also the maximizing $P_{\tau}$ is given by

$$
P_{\tau}^{*}\left(T_{d}\right)=\frac{P T \sqrt{\left(T_{d}+P T\right) M}}{\left(T-T_{d}\right)\left(\sqrt{\left(P T+T_{d}\right) M}+\sqrt{(M+P T) T_{d}}\right)}
$$

In order to maximize the achievable rate over $T_{d}$ we have to solve the following optimization problem

$$
R^{\star}=\max _{T_{d}, 0 \leq T_{d} \leq T-M} \frac{T_{d}}{T} \mathrm{E}_{\left\{g_{i}\right\}} f\left(P_{\mathrm{eff}}^{\star}\left(T_{d}\right)\right)
$$

where $P_{\mathrm{eff}}^{\star}\left(T_{d}\right)$ is given in (12) and $f(x)$ is defined as

$$
f(x)=\max _{p_{i}, \sum_{i} p_{i} \leq 1} \log \left|I+x \sum_{i=1}^{n} p_{i} g_{i}^{*} g_{i}\right|
$$

It is shown in [14] that the cost function in (14) is increasing in $T_{d}$. Therefore the optimal $T_{d}$ is $T-M$. The next theorem summarizes the above arguments.

Theorem 5: Consider a block fading MISO broadcast channels with $M$ transmit antennas, coherence interval of $T \geq M$ and total transmit power of $P$. Further assume that the channel coefficients are independent zero mean unit variance Gaussian random variables. The following sum-rate is achievable using training

$$
R^{\star}=\frac{T-M}{T} \mathrm{E}_{\left\{g_{i}\right\}} \max _{p_{i}, \sum_{i} p_{i} \leq 1} \log \left|I+P_{\text {eff }}^{\star}(T-M) \sum_{i=1}^{n} p_{i} g_{i}^{*} g_{i}\right|
$$

where $P_{\text {eff }}^{\star}(\cdot)$ is defined in (12). Furthermore this rate is achieved by using orthogonal and fixed power training vectors over the first $M$ time samples and transmitting data over the remaining portion of the coherence interval. The power of each training vector is $P_{\tau}^{\star}(T-M)$ and is given in (13).

The following Corollary gives further insights on the behavior of the sum-rate in different regimes.

Corollary 1: Consider the MISO broadcast channel model described in Theorem 5. Then the achievable sum-rate

- For large $P$ scales like

$$
R^{\star}=\min \{M, n\}\left(1-\frac{M}{T}\right) \log P .
$$

- For small $P$ scales like

$$
R^{\star}=\frac{T c \log e}{4 M} P^{2}
$$

where $c$ is the mean of the maximum of $n$ i.i.d random variables with $\chi^{2}(2 M)$ distribution.

- For large number of users $(n)$ scales like

$$
R^{\star}=M\left(1-\frac{M}{T}\right) \log \left(1+P_{\text {eff }}^{\star}(T-M) \log n\right)
$$

In particular, Corollary 1 shows that using training-based schemes one can achieve the multiplexing gain of a MIMO pointto-point channel with $M$ transmit and $n$ receive antennas in the high SNR regime. However the power invested in the training phase increases linearly with $P$ (see (13) for large $P$ ). Also the required feedback rate for sending the estimates to the transmitter should increase with $P$. 


\section{CONCLUSION}

This paper considers the effect of channel estimation error on the capacity region of MIMO Gaussian broadcast channels. An achievable rate region based on dirty paper coding is derived. It is further shown that for MISO case this region is equivalent to the capacity region of a multi-access channel with noise covariance matrix that depends on the transmit power and the estimation error. A training-based scheme for block fading MISO Gaussian broadcast channels is analyzed and the optimal length of training interval and the power used for training is derived. Designing practical schemes in the presence of channel estimation error is an important future work. Also finding outer bounds on the capacity region of broadcast channels with estimation error is an interesting problem (see [17]).

\section{APPENDIX A}

In this section we sketch the proof of Theorem 3. We will use the approach taken in [7]. Instead of looking at the achievable rate, we will look at the feasibility of a set of SINR constraints in the broadcast and the dual multi-access scenario. Similar to [8], we consider beam-forming with dirty paper precoding. The transmitted signal in this case can be written as

$$
x=\sum_{i} W_{i} u_{i}
$$

where $W_{i}$ is the $i$-th beam that carries $u_{i}$ the information for user $i$. Without loss of generality we assume that $\mathrm{E}\left|u_{i}\right|^{2}=1$. Looking back at the definition of $B_{i}$ in Theorem 1 , here we have assumed that $B_{i}=W_{i} W_{i}^{*}$. Next we consider the problem of minimizing total transmit power subject to a set of SINR constraints for broadcast channel

$$
\begin{array}{rl}
L^{B C}=\min _{P, w_{i}} & P \\
\text { subject to } & \frac{\left|h_{i} w_{i}\right|^{2}}{\sum_{j>i}\left|h_{i} w_{j}\right|^{2}+1+\sum_{l=1}^{n} w_{l}^{*} A_{i} w_{l}} \geq \gamma_{i} \\
\sum_{i=1}^{n} w_{i}^{*} w_{i} & \leq P .
\end{array}
$$

Similarly we can write the following problem for the dual multiple access channel. The SINR's shown below are achieved by using $v_{i}$ as a filter for $i$-th user information and using interference cancelation.

$$
\begin{aligned}
L^{M A} & =\min _{P_{i} \geq 0, v_{i}} \sum_{i=1}^{n} P_{i} \\
& \text { subject to } \frac{P_{i}\left|h_{i} v_{i}\right|^{2}}{\sum_{j<i}\left|h_{j} v_{i}\right|^{2}+v_{i}^{*}\left(\sum_{l=1}^{n} P_{l} A_{l}\right) v_{i}} \geq \gamma_{i}
\end{aligned}
$$

Following the steps of [8], we can show that both of the above problems have the following dual

$$
\begin{aligned}
\max _{P_{i} \geq 0} & \sum_{i=1}^{n} P_{i} \\
\text { subject to } & \sum_{j<i} P_{j} h_{j}^{*} h_{j}+\sum_{l=1}^{n} P_{l} A_{l}+I \succeq \frac{P_{i}}{\gamma_{i}} h_{i}^{*} h_{i}
\end{aligned}
$$

Furthermore the strong duality holds and the two problems have the same minimum power and are equivalent. Therefore the achievable rate region of MISO broadcast channel is equivalent to the capacity region of a multiple access channel with $M$ antennas at the receiver, total power constraint $P$ and a noise covariance $Q_{\text {eq }}$ that depends on the different users' transmit powers in the following form

$$
Q_{\text {eq }}=I+\sum_{l=1}^{n} P_{l} A_{l},
$$

and this proves Theorem 3 .

\section{REFERENCES}

[1] G. Caire and S. Shamai (Shitz), "On the achievable throughput of a multiantenna Gaussian broadcast channel," IEEE Trans. on Info. Theory vol. 49, no. 7, pp. 16911706, Jul. 2003.

[2] P. Viswanath and D. N. C. Tse, "Sum capacity of the vector Gaussian broadcast channel and downlink-uplink duality," IEEE Trans. Inf. theory, vol. 49, no. 8, pp. 19121921, Aug. 2003.

[3] S. Vishwanath, N. Jindal, and A. Goldsmith, "Duality, Achievable Rates, and Sum-Rate Capacity of Gaussian MIMO Broadcast Channels," IEEE Trans. on Info. Theory, Volume 49, Issue 10, pp. 2658-2668, Oct. 2003.

[4] Wei Yu, and John Cioffi, "Sum Capacity of Gaussian Vector Broadcast Channels," IEEE Trans. on Info. Theory, vol. 50, no. 9, pp.1875-1892, Sept. 2004.

[5] H. Weingarten, Y. Steinberg, and S. Shamai (Shitz), "The capacity region of the Gaussian MIMO broadcast channel," Proceedings of Conference on Information Sciences and Systems, Mar. 2004.

[6] M. Costa, "Writing on dirty paper," IEEE Trans. Inform. Theory, vol. 29 , no. 3, pp. 439441, May 1983.

[7] Wei Yu, Tian Lan, "Transmitter Optimization for the Multi-Antenna Downlink with Per-Antenna Power Constraints," submitted to the IEEE Trans. on Signal Processing, Dec. 2005

[8] Wei Yu, "Uplink-Downlink Duality via Minimax Duality," IEEE Trans. on Info. Theory, vol. 52, no. 2, Feb. 2006.

[9] D. Tse and S. Hanly, "Multi-Access Fading Channels: Part I: Polymatroid Structure, Optimal Resource Allocation and Throughput Capacities", IEEE Transactions on Information Theory, vol. 44, no. 7, Nov., 1998, pp. 27962815 .

[10] S. Gelfand and M. Pinsker, "Coding for channel with random parameters,"Probl, Control Info. Theory, vol.9, no. 1, pp. 19-31, Dec. 1980.

[11] T. Y. Al-Naffouri, M. Sharif, and B. Hassibi, "How much does transmit correlation affect the sum-rate of MIMO downlink channels?,"submitted to IEEE Trans. on Wireless Comm, 2006.

[12] M. Medard, "The effect upon channel capacity in wireless communication of perfect and imperfect knowledge of the channel," IEEE Trans. Info. Theory, vol. 46, pp. 933946, May 2000.

[13] B. Hassibi and B.M. Hochwald, " How much training is needed in a multiple-antenna wireless link?," IEEE Trans. on Info. Theory, vol.49, no.4, Apr. 2003, pp. 951-964.

[14] A. F. Dana, M. Sharif, and B. Hassibi, "On the Capacity Region of Multi-Antenna Gaussian Broadcast Channels with Estimation Error," in preparation for submission.

[15] M. Sharif and B. Hassibi, "On the capacity of MIMO broadcast channels with partial side information," IEEE Trans on Info. Theory, vol. 51, Feb. 2005 .

[16] M. Sharif, A. F. Dana, A. Vakili, and B. Hassibi,"Differentiated rate scheduling for the downlink of cellular system," to be submitted to IEEE Trans. on Comm, 2006.

[17] A. Lapidoth, S. Shamai, and M. Wigger, "On the capacity of a mimo fading broadcast channel with imperfect transmitter side-information," Proceedings of Allerton Conf. on Commun., Control, and Comput., Sept. 2005.

[18] B. Hochwald, B. Peel, and A. L. Swindlehurst, "A Vector perturbation technique for near capacity multiantenna multiuser communication-Part II: Perturbation," IEEE Trans. Comm., no. 3, pp. 537-545, March 2005.

[19] S. A. Jafar and A. J. Goldsmith, "Multiple-Antenna capacity in correlated Rayleigh fading with channel covariance information", IEEE Transactions on Wireless Communications, vol. 4, no. 3, pp. 990-997, May 2005.

[20] T. Yoo and A. Goldsmith, "On the Optimality of Multi-Antenna Broadcast Scheduling Using Zero-Forcing Beamforming" To appear: IEEE JSAC Special Issue on $4 G$ Wireless Systems.

[21] M. Kobayashi, G. Caire, D. Gesbert, "Opportunistic beamforming vs. space time coding in a queued downlink," 14th IST Mobile and Wireless Communications Summit, June 19-23, 2005, Dresden, Germany. 